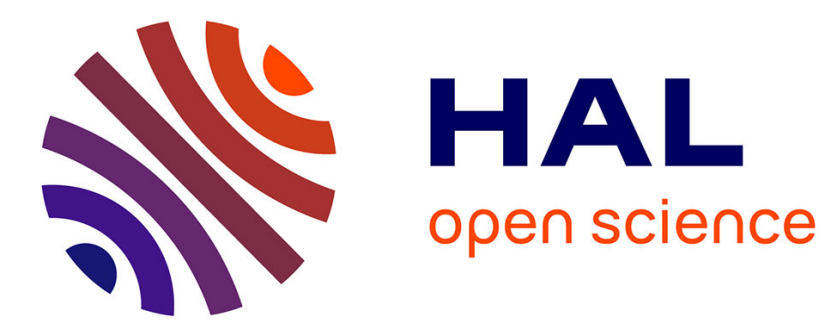

\title{
Dynamic analysis of nonlinear elastic materials
}

Christian Wielgosz, Gilles Marckmann

\section{- To cite this version:}

Christian Wielgosz, Gilles Marckmann. Dynamic analysis of nonlinear elastic materials. Computational Materials Science, 1996, 7 (1-2), pp.1-4. 10.1016/S0927-0256(96)00051-1 . hal-01391003

\section{HAL Id: hal-01391003 \\ https://hal.science/hal-01391003}

Submitted on 3 Nov 2016

HAL is a multi-disciplinary open access archive for the deposit and dissemination of scientific research documents, whether they are published or not. The documents may come from teaching and research institutions in France or abroad, or from public or private research centers.
L'archive ouverte pluridisciplinaire HAL, est destinée au dépôt et à la diffusion de documents scientifiques de niveau recherche, publiés ou non, émanant des établissements d'enseignement et de recherche français ou étrangers, des laboratoires publics ou privés. 


\title{
Dynamic analysis of nonlinear elastic materials
}

\author{
C. Wielgosz ${ }^{\mathrm{a}, *}$, G. Marckmann ${ }^{\mathrm{b}, 1}$ \\ ${ }^{a}$ Fuculté des Sciences et des Techniques, 2 rue de la Houssinière, 44072 Nantes Cedex, France \\ ${ }^{\mathrm{b}}$ Ecole Centrale de Nantes, 1 rue de la Noë, 44072 Nantes Cedex. France
}

\begin{abstract}
The paper is concerned with the programmation of the constitutive law of hyperelastic materials so as to compute their response to impact loading. The law is obtained from a Harth-Smith model for a given strain rate. A mixed variational principle is used to develop a finite element program for 3D dynamic behavior of nearly incompressible materials.
\end{abstract}

Keywords: Hyperelasticity; Impact; Mixed method; Dynamic

\section{Dynamic constitutive law of hyperelastic mate- rials}

This kind of materials is very difficult to study. The main difficulties are due to many facts: these materials are nonlinear and hyperelastic, they can have very large strains (up to $500 \%$ in compression and more that $50 \%$ in traction), they are incompressible or nearly incompressible and very sensitive to the strain rate. The construction of the constitutive law has been done from experimental results performed on a sample for different values of the strain rate $\dot{\varepsilon}[1]$.

We suppose that the sample is submitted to a constant average value of the strain rate depending on the impact velocity $V$ (Fig. 1).

Piola-Kirchoff stresses $\sigma$ are assumed to be independent of the history of the material and to

\footnotetext{
"Corresponding author. Tel:: +33-240373180; fax: + 33240140048; e-mail: wielgosz@physique.univ-nantes.fr.

'Tel.: + 33-240372514; fax: + 33-240747406; e-mail: marckmann@ec-nantes.fr.
}

derive from the internal energy $Q\left(I_{1}, I_{2}, I_{3}\right)$, where $I_{1}, I_{2}, I_{3}$ are the three invariants of the right Cauchy-Green tensor $C$. One can find that:

$\sigma=2\left\{\left[\frac{\partial Q}{\partial I_{1}}+I_{1} \frac{\partial Q}{\partial I_{2}}\right] I-\frac{\partial Q}{\partial I_{2}} C+\frac{\partial Q}{\partial I_{3}} \operatorname{cof} C\right\}$

where $I$ is the unit tensor, and $\operatorname{cof} C$ is the matrix of cofactors of $C$.

A Hart-Smith law has been chosen to describe $Q$ and to obtain the constitutive relations:

$$
\begin{aligned}
Q\left(I_{1}, I_{2}, I_{3}\right)= & c_{1} \int \exp \left[c_{3}\left(I_{1}-3\right)^{2}\right] \mathrm{d} I_{1} \\
& +3 c_{2} \ln \left(I_{2}\right)+c_{4}\left(I_{3}-1\right) \\
& +\frac{1}{2} c_{5}\left(I_{3}-1\right)^{2}
\end{aligned}
$$

In our model, the coefficients $c_{i}(i=1, \ldots, 5)$ are supposed to be constant for an average strain rate $\dot{\varepsilon}$.

The parameter $c_{4}$ depends on the other parameters and is obtained by writing that the stresses are equal to zero in the undeformed state:

$c_{4}=-\left(c_{1}+2 c_{2}\right)$ 

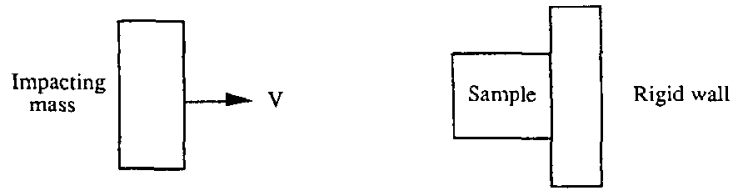

Fig. 1.

If we compare now a compressible rubber-like material with a classical elastic material at low strain, we have the following relations:

$G=2\left(c_{1}+c_{2}\right), \quad c_{5}=\frac{c_{1}(1+\nu)+3 c_{2}(1-\nu)}{3(1-2 \nu)}$

where $G$ is the shear modulus and $\nu$ is the Poisson's ratio.

For nearly incompressible materials we notice that $c_{5}$ is very large, for incompressible materials $\left(I_{3}=1\right.$ and $\nu=0.5) \quad c_{5}$ becomes infinite and $\partial Q / \partial I_{3}$ is undetermined. In such a case, a Lagrange multiplier $p$ can be introduced to take in account the incompressibility condition [2]:

$p=\left(\frac{\partial Q}{\partial I_{3}}\right)_{I_{3}=1}$ and $\quad Q=Q\left(I_{1}, I_{2}\right)+p\left(I_{3}-1\right)$

For a sample in static loading, the unknown multiplier $p$ can be eliminated from the equilibrium equations by writing the boundary conditions. The Piola-Kirchoff stresses $\sigma$ are then easily obtained from the internal energy. The deduced Cauchy stresses are then used to calibrate the law. For instance, a traction-compression load is governed by the following equation:

$$
\begin{aligned}
\frac{F}{S}= & 2 \lambda\left(\lambda-\frac{1}{\lambda^{2}}\right)\left[c_{1} \exp \left(c_{3}\left(\lambda^{2}+\frac{2}{\lambda}-3\right)\right)\right. \\
& \left.+\frac{\lambda c_{2}}{\left(1+2 \lambda^{3}\right)}\right]
\end{aligned}
$$

where $\lambda=l / l_{0}$ is the elongation of the sample, $F$ is the measured force, and $S$ is the section in the deformed state.

This example shows how the parameters $c_{i}$ may be identified. In fact, a traction-compression test is not sufficient.

We now consider that the parameters are given and constant during the impact at a nonzero strain rate $[1]$

\section{Finite element implementation}

The virtual work principle, written in the total Lagrangian formulation, gives:

$$
\begin{aligned}
& \forall \delta u \quad \int_{\Omega}-\delta \varepsilon^{T} \cdot \sigma \mathrm{d} \Omega+\int_{\partial \Omega} \delta u \cdot T \mathrm{~d} \Omega \\
& \quad+\int_{\Omega} \delta u \cdot f \mathrm{~d} \Omega \\
& \quad=\int_{\Omega} \delta u \cdot \rho \ddot{u} \mathrm{~d} \Omega
\end{aligned}
$$

where $\Omega$ denotes the undeformed state, $\varepsilon$ is the Green-Lagrange strain, $\sigma$ is the Piola-Kirchoff stress, $T$ denotes surface forces on $\partial \Omega$, and $f$ denotes body forces. Unfortunately, this principle cannot be directly used for any incompressible continuum because the stress $\sigma$ is dependent on $p$ which is unknown in the constitutive law. We have, therefore, to use a penalty method to impose $I_{3}=1$ to a compressible material:

$p=c_{4}+c_{5}\left(I_{3}-1\right)$

where $c_{5}$ is a high modulus and $c_{4}$ is the value of $p$ in the undeformed state. It is quite similar as to consider the material as compressible with a high compressibility modulus.

The problem is then solved with a mixed variational formulation. A mixed method has been often used in static analysis of rubber-like materials [2-4]. In case of dynamic analysis a direct implicit finite element method was introduced by Barsoum [3]. A dynamic mixed f.e. is here developed, it seems for the first time. The incompressibility condition is now replaced by the variational equation

$$
\forall \delta p \quad \int_{\Omega} \delta p\left(I_{3}-1-\frac{1}{c_{5}}\left(p-c_{4}\right)\right) \mathrm{d} \Omega=0
$$

The field $p$ is computed at each time step over all finite elements and the stress is finally obtained by the constitutive relations. The first variational equation is then solved by the well known explicit finite element method (with a central finite differences scheme) to compute the displacement field. The computational time step is very dependent on the pseudo-compressible-modulus $c_{5}$ and must be very small. 


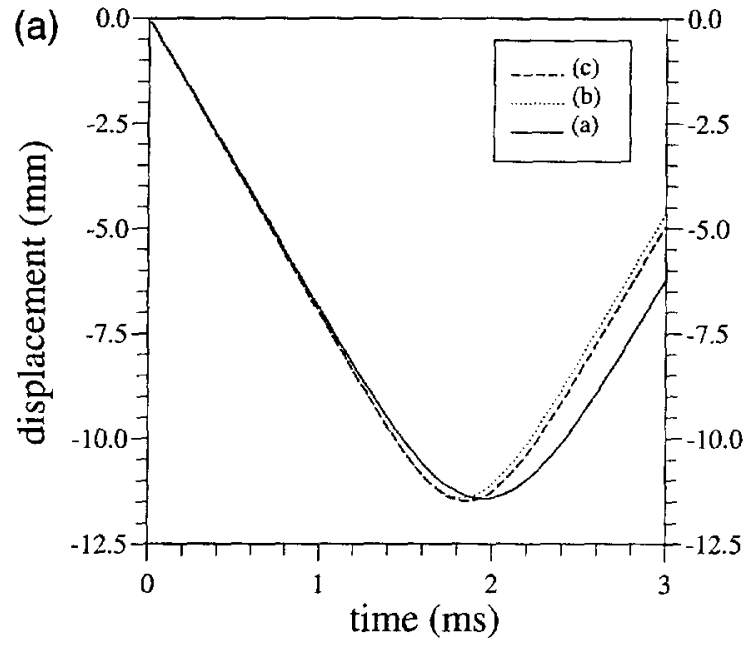

displacement of impacted nodes

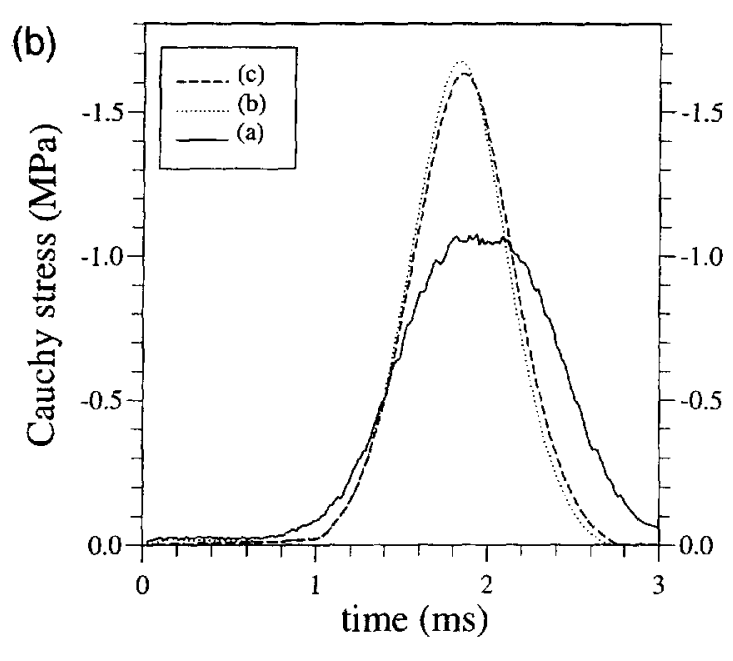

stress in impacted sample

Fig. 2.

The impacted sample is then discretized with 8 nodes volume finite elements. Numerical results are compared to experiments. There is a good agreement for the displacement field and for the mean values of the stresses, but these results contain many oscillations which provide from the penalty method used to solve the second variational equation. A prediction-correction process on the value of $p$ is, therefore, introduced to eliminate oscillations. The method will be named a modified explicit method (M.E.M).

\section{Numerical results}

Numerical results are performed on the impacted sample discretized in volume finite elements. Result (a) is compared to a one dimension finite element calculation (b). A third computation (c) is made with a volume finite element model where the mass of the material is neglected in order to approach the one dimensional model with no inertia in lateral directions (Fig. 2).

The agreement between (b) and (c) means that our

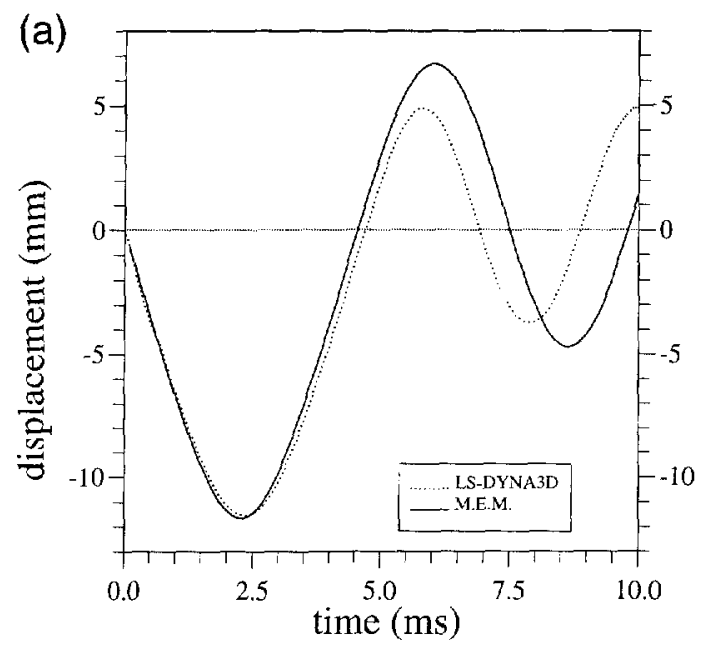

comparison of displacements

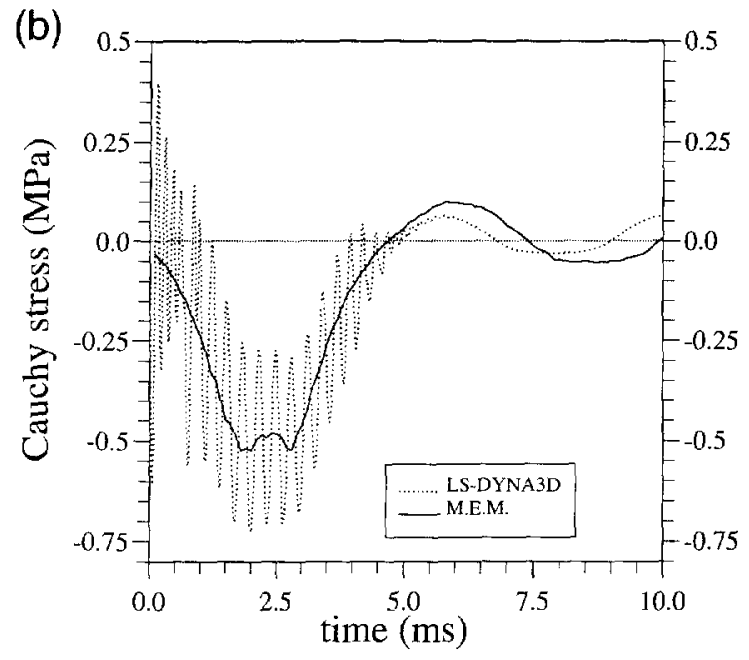

comparison of Cauchy stresses

Fig. 3. 
program and our method are accurate and this allows also to conclude that the difference between (c) and (a) is due to the inertia and especially the own mass of the rubber-like material. This must be taken into account to calibrate the constitutive law.

Our results are also compared with LS-DYNA3D [5] for a rubber sample submitted to an impacting mass. The constitutive law used in this program provides from a Mooney-Rivlin model. One can see on Fig. 3 that the classical explicit f.e.m. used in LS-DYNA3D gives many oscillations on the stresses and that these oscillations have almost disappeared with our M.E.M.

We have also proved that incompressible materials can be analyzed using the central difference method, which was denied by Barsoum [3].

\section{Conclusion}

A mixed variational principle was used to develop a finite element program for 3D dynamic behavior of nearly incompressible materials. Numerical results show that the program is accurate for a given strain rate. The following step of the study will be to fit the constitutive law for each strain rate and to take into account its evolution during the impact. This approach is an alternative to avoid the visco-elasticity theory.

\section{References}

[1] M. Dannawi and C. Wielgosz, Charactérisation dynamique, modélisation numérique des simulants élastiques, Rapport de contrat DGA No. 25122/93/CEB/DED/PB (1994).

[2] M. Jazzar, Thè̀se, Université d'Aix-Marseille II (1993).

[3] E.G. Barsoum and M.P. Bieniek, AIAA J. 93-1327-CP (1993) 273-281.

[4] T. Schamhost and T.H.H. Pian, Int. J. Num. Met. Eng. 12 (1978) 665-676.

[5] LS-DYNA3D, Livermore software technology (1995). 